\title{
Spezifische Immuntherapie wirkt kausal
}

$E_{\text {in }}^{\text {in }}$ ine der tragenden Säulen in der Behandlung des allergischen Asthma bronchiale ist die frühzeitige spezifische Immuntherapie (SIT), die es seit etwa 50 Jahren gibt. Die mit standardisierten AllergenPräparaten vorgenommene SIT ist die einzige Möglichkeit, den natürlichen Krankheitsverlauf kausal zu beeinflussen. Die SIT drängt auf lange Sicht nicht nur die Symptomatik zurück, sondern vermag darüber hinaus die Progredienz allergischer Atemwegsleiden aufzuhalten.

Langfristig bilden sich dank SIT die allergenspezifische und unspezifische nasale und bronchiale Hyperreaktivität zurück. Es kommt zu einer über Jahre oder sogar lebenslang anhaltenden Immunmodulation zugunsten der $\mathrm{TH} 1$ Immunantwort sowie zu einer Toleranzinduktion. Dadurch sind die Patienten bei erneutem Allergenkontakt vor einer akuten Verschlechterung der Lungenfunktion geschützt, versicherte Prof. K.-C. Bergmann, Bad Lippspringe.

\section{Geringeres Risiko von Neu-}

\section{sensibilisierungen}

Die mit modernen und molekular standardisierten Allergen-Präparaten subkutan durchgeführte SIT senkt beim pollenallergischen Asthmatiker die asthmatischen wie rhinokonjunktivalen Symptome um 90\%, und der Medikamentenverbrauch geht um bis zu $80 \%$ zurück. Patienten, die auf Hausstaubmilben allergisch reagieren, profitieren gleichfalls von der SIT, die überdies das Risiko von Neusensibilisierungen eindämmt. Kontraindiziert ist das Verfahren, wenn trotz adäquater Pharmakotherapie schweres Asthma besteht und der $\mathrm{FEV}_{1}$ unter $70 \%$ des Sollwerts liegt.

„Das Prinzip der sublingualen Immuntherapie ähnelt dem der spezifischen Immuntherapie. Allerdings werden die Allergen-Extrakte nicht subkutan injiziert, sondern vom Patienten täglich unter die Zunge getropft, in der Regel über drei Jahre“, erläuterte Dr. H. Wolf, Hamburg. Die tägliche Allergen-Aufnahme durch die Langerhans-Zellen der Mund- mukosa sorgt für einen raschen Wirkeintritt.

SLIT One, bei dem das Tropfenzählen entfällt, ist eine Weiterentwicklung der sublingualen Immuntherapie mit Pangramin SLIT. Wird die sublinguale Immuntherapie in der symptomfreien Zeit gestartet, sind auch hohe Anfangsdosen sicher und gut verträglich. Deshalb lässt sich die Initialphase, also die Aufdosierung bis zur Erhaltungsdosis, auf zehn Tage verkürzen. Wer unter Gräserpollenallergie leidet, kann deshalb noch kurz vor dem Pollenflug mit der sublingualen Immuntherapie beginnen und erfreut sich schon in der aktuellen Gräserpollensaison einer deutlichen Rückbildung der Beschwerden.

K. B. Filip, Landsberg am Lech

Quelle: Satellitensymposium „Neues zur spezifischen Immuntherapie (SIT+SLIT) bei allergischen Atemwegserkrankungen" anlässlich des 45. Kongresses der Deutschen Gesellschaft für Pneumologie, März 2004 in Frankfurt a. M., Veranstalter: Alk-Scherax, Hamburg. 